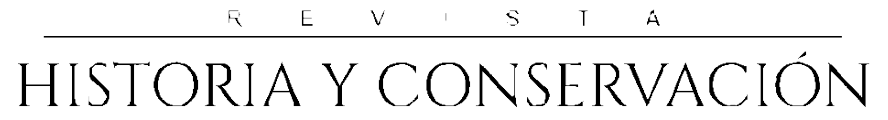 \\ DEL PATRIMONIO EDIFICADO
}
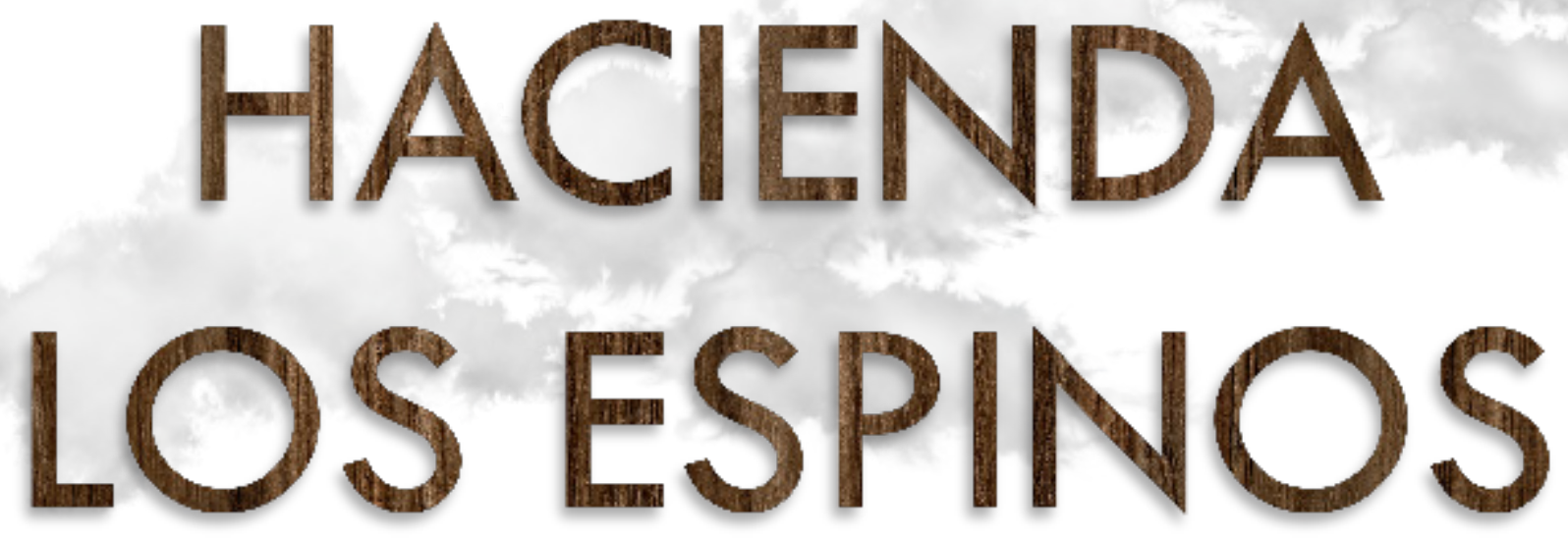


\section{HACIENDA LOS ESPINOS. ORIGEN Y TRASCENDENCIA.}

HACIENDA LOS ESPINOS. ORIGIN AND TRANSCENDENCE. Juan Carlos Camacho Guzmán

Fecha de envío: 25 de agosto de 2018

Fecha de aceptación: 20 de septiembre de 2018

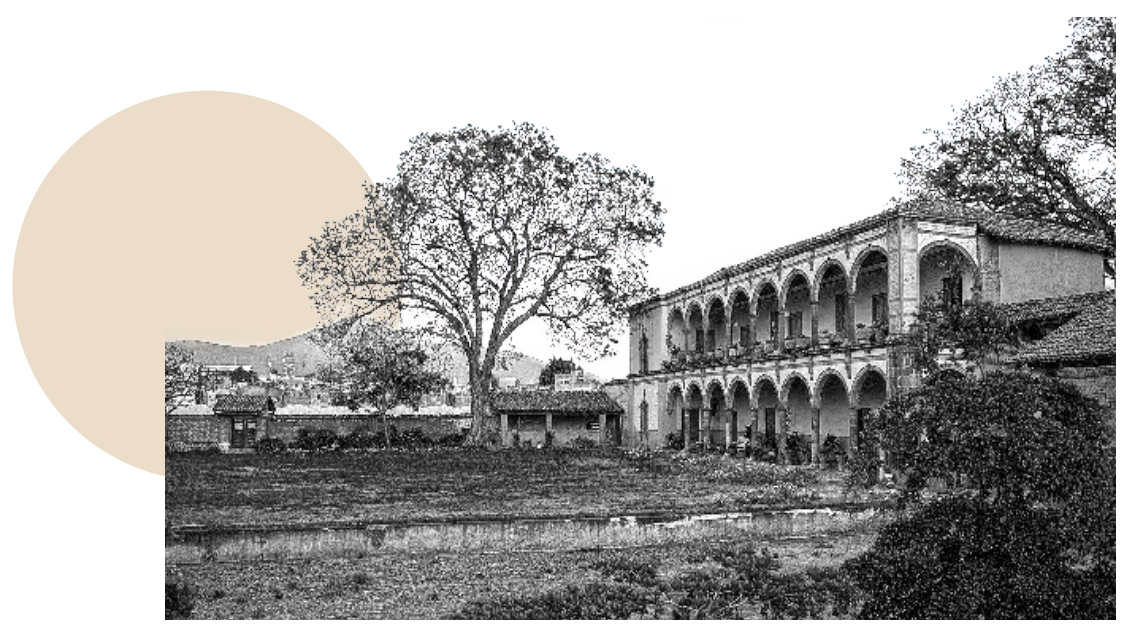

- Resumen:

Los procesos históricos que conducen al origen del territorio de la hacienda Los Espinos relatan el rumbo del desarrollo de una comunidad agrícola singular. Los valores patrimoniales que la historia materializa en los vestigios arquitectónicos del casco hacendario son dignos de preservar.

Debido a la evidente unión de linderos entre la hacienda y la villa de Zamora, se podría indicar que la ciudad tuvo su origen en este complejo productivo, donde su población se desarrolló a partir de la comunidad de labores que la habitaban. Sin embargo, los antecedentes refutan esta teoría.

Se muestra un recorrido por los antecedentes territoriales del asentamiento hacendario, además de reconocer el proceso de establecimiento, apropiación, organización y ordenamiento territorial, para estudiar en qué medida influyeron las relaciones establecidas de los propietarios con las autoridades civiles y eclesiásticas para la obtención, consolidación y pérdida de la arquitectura de la hacienda y su territorio.

\section{Palabras Clave:}

Asentamiento, Hacienda, merced, patrimonio, territorio, Zamora.
- Summary:

The historical processes that lead to the origin of the territory of the hacienda Los Espinos relate the course of the development of a unique agricultural community. The patrimonial values that history materializes in the architectural vestiges of the hacienda are worthy to be preserved.

Due to the evident union of boundaries between the hacienda and the town of Zamora, it could indicate that the city had its origin from this productive complex, where its population was developed from the community of work that inhabited it. However, the antecedents refute this theory.

It shows a tour of the territorial background of the hacendario settlement, in addition to recognizing the process of establishment, appropriation, organization and territorial ordering, studying to what extent the established relations of the owners with the civil and ecclesiastical authorities for the obtaining, consolidation and loss of the architecture of the hacienda and its territory.

\section{Key words:}

Settlement, property, mercy, heritage, territory, Zamora. 


\section{INTRODUCCIÓN}

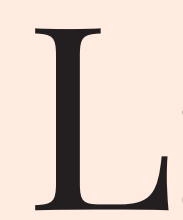

La explicación trasciende desde el antecedente de este asentamiento hacendario virreinal con una relación directa en el desarrollo de la villa de Zamora. A través de una serie de cambios en la propiedad territorial y las relaciones de parentesco de las familias de alto capital económico involucradas, se permite la comunión con cargos políticos, recursos sociales, negociaciones religiosas, acumulación de múltiples propiedades y la implementación de tecnología hidráulica necesaria para la explotación y comercio.

La historia de la hacienda Los Espinos proviene de la familia de los Jasso, desde sus generaciones de la conquista, y se establece posterior a la fundación de la villa en 1574, hipotéticamente a finales del siglo XVI y principios del XVII. Esta inferencia se basa en la explicación de la formación de la hacienda y la vida económica durante la época colonial que nos brinda María Teresa Jarquín en La formación de la hacienda y la vida económica (2004), con lo que es posible construir un panorama que explique el desarrollo de Los Espinos. 


\section{ANTECEDENTES DEL} TERRITORIO

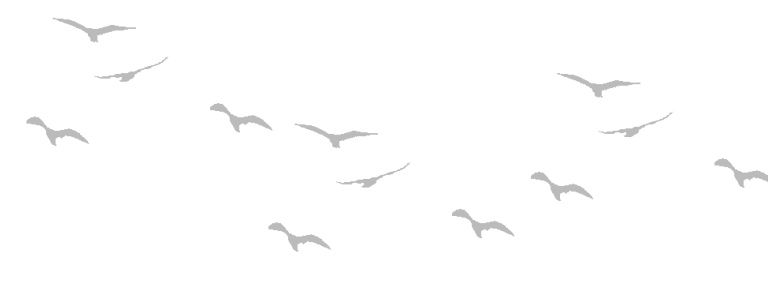

Las primeras poblaciones del Nuevo Mundo y la vida económica se vieron afectadas por epidemias que causaron devastaciones en $1531,1545,1564$, entre otros años, lo que empeoró considerablemente la mortalidad entre los indigenas hasta el punto de acabar con comunidades enteras en los sitios de mayor población. Los asentamientos más afectados necesitaron vender sus tierras para pagar los tributos reales. El aprovechamiento de la situación de los caciques motivó la invasión de terrenos, amparados con compras ficticias o asegurando que se trataba de sitios abandonados, para posteriormente ofrecerlos a los españoles (Jarquín, 2004).

Desde un inicio, la economía de los pueblos alrededor del bajío zamorano se desarrolló principalmente en lo que fue el valle nominado por los tarascos Tzirondaro, o Cirándaro, que significa "lugar de ciénegas"; otros argumentan que fue llamado por los mismos purépechas como Xuenguagueo, o que los indios tecos lo Ilamaban Huenguagueo, que en español sería "lugar de verdura" (Rodríguez, 1952).

A la llegada de la colonia española, no solo se redimensionaron las estructuras en los ámbitos militares, políticos, económicos y sociales de Michoacán, sino que también el territorio sufrió modificaciones considerables. Primordialmente la trascendencia en la integración de extensas estancias de producción, incluyendo el impacto de la ganadería en la comarca, transformó a la región en un abastecedor clave de productos necesarios para la explotación de la minería en la Nueva España, a la par de las campañas de conquista y colonización del septentrión novohispano. 
Sin embargo, el distrito de Jacona, una nueva población de cabecera abanderada por los agustinos en comunión con los naturales, mantuvo su esencial base en la agricultura en los linderos de lo que posteriormente fue la villa de Zamora. En sus periodos de adquisición territorial a base de encomiendas y mercedes, comenzó a albergar vecinos españoles que antecedieron la consolidación de una organización en unidades productivas: las denominadas haciendas, modelo de propiedad del territorio y una de las riquezas y poderío más prestigiosas en el centro de la Nueva España desde finales del siglo XVI, las cuales conformaron el antecedente del asentamiento primigenio de muchas poblaciones.

El establecimiento originario que antecede a Los Espinos radica en lo que fue la provincia de Xacona, la cual se componía de varios poblados, entre los que se encontraron a Ichaparaco e Itúcuaro, Iztán o Iztlán, donde eventualmente se avecinaron más españoles con mercedes otorgadas antes de la fundación de Zamora. De las mercedaciones que se aproximan a la descripción del territorio hacendario en cuestión, se encuentra la otorgada a Bartolomé Castañón de Aguerón consistente en un sitio para ganado mayor con dos caballerias de tierra para el sustento, en términos de dicho pueblo de Xacona el 13 de junio de 1567 (Rodríguez, 1952).

El Río Duero, se convirtió en un protagonista esencial que definió los límites del asentamiento de la villa y de la hacienda, por ser barrera natural y proveedor del recurso vital. En la fundación, el río dividió las tierras al norte para los españoles en la salida del camino a Ixtlán y al sur para los naturales con los linderos de la actual Jacona.

Por esta condicionante, se propiciaron invasiones territoriales indebidas de los habitantes españoles hacia el sur, como se reportan para 1689 los casos de Inés de Luna, José Lupiana, Miguel Ochoa y Antonio Vejines. Sin embargo, los tecos fueron amparados. Es sencillo pensar que las tierras colindantes con este río fueron codiciadas por su potencial recurso, además de notarse ese reflejo en las descripciones de las mercedes otorgadas después de la fundación de la villa.

Dichas descripciones no aclaran con precisión los territorios asignados, por lo que existe una gran lista de pobladores que fueron beneficiados con tierras cerca del río, lo cual resulta en una indeterminación al tratar de localizar cuál de las mercedes perteneció a los terrenos de la hacienda. Aunado a esto, las mercedes otorgadas durante la fundación y posteriores a esta se limitaron a ser solares o estancias de caballerías dentro de la traza de la villa, contrario a las primeras encomiendas o sitios antecesores de ganado mayor y menor. De manera que, sin pruebas contundentes, se descarta el hecho de que el extenso patrimonio de Los Espinos se haya determinado en la época del nacimiento de la villa zamorana (ver figura 1 ). 


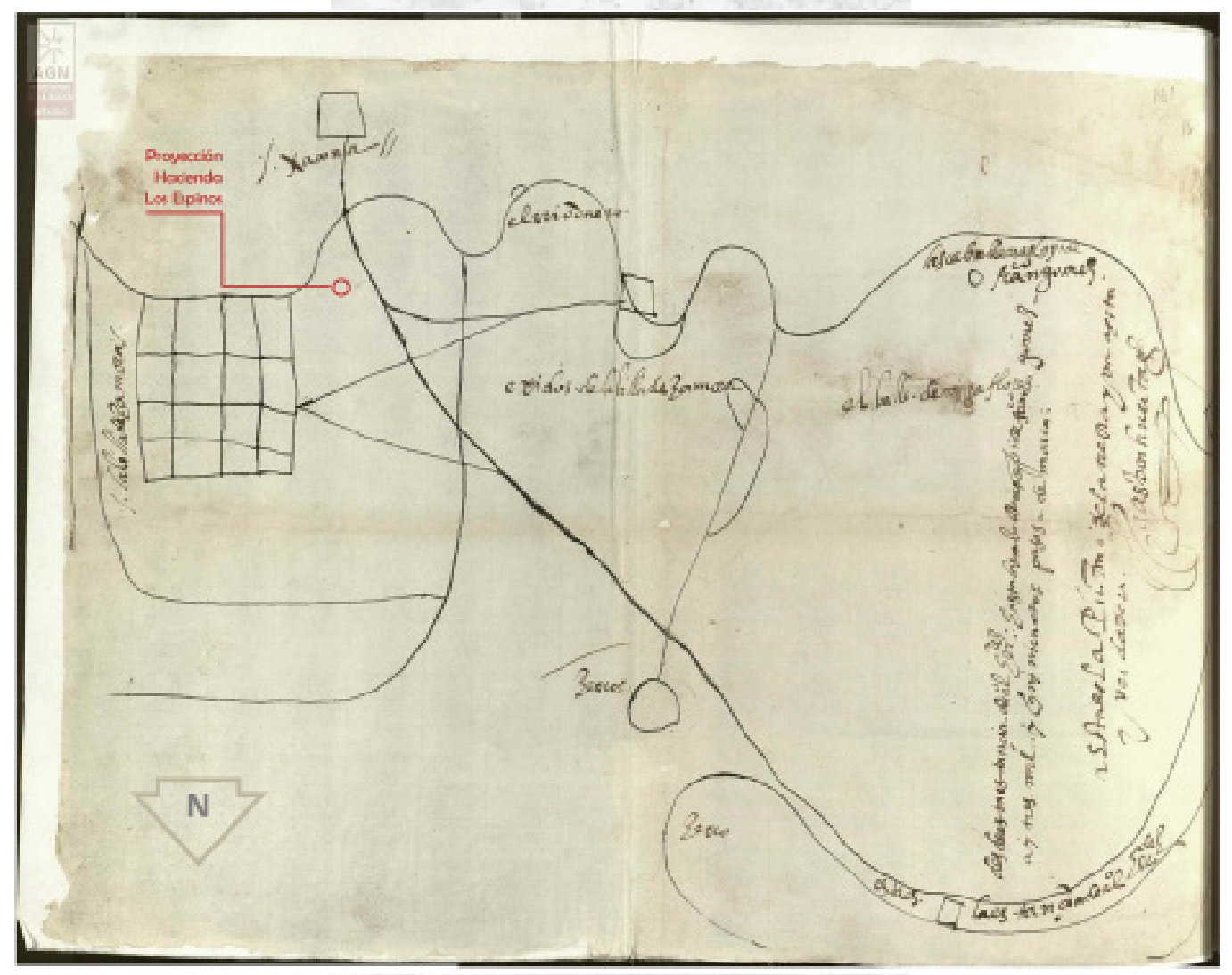

Figura 1. La Villa de Zamora a breve distancia de Jacona y a una gran lejanía del valle de Miraflores.

- Proyección de la hacienda Los Espinos. En el documento aparecen unos cerros, la estancia de Alonso Hernández, así como el río. Francisco Gómez, vecino de la villa de Zamora, pidió de merced dos caballerías de tierra, en términos de dicha villa. En algunas de las primeras mercedes otorgadas por la Corona a los vecinos de Xacona, recientes a la fundación de Zamora de 1574, existieron múltiples cambios en la propiedad, abandono de tierras, ventas y herencias posteriores. Arturo Rodríguez Zetina comenta que llegó a existir en la caja del cabildo del ayuntamiento la traza de la villa conforme a las mercedes otorgadas con los nombres de los propietarios en ese entonces.

Gaspar Huerta, Xacona; Zamora. Mich., 1583, número de pieza: 2096, clasificación 978/0735. Referencia: Tierras, vol. 2769, exp. 9, fs. 160 v y 161, Archivo General de la Nación. 
Por lo tanto, la historia de la hacienda Los Espinos proviene de la familia de los Jasso en sus generaciones desde la Conquista. Se establece a finales del siglo XVI y principios del XVII, posteriormente a la fundación de la villa. La inferencia se apoya en la explicación de la formación de la hacienda y la vida económica durante la época colonial, con lo que es posible construir un panorama que explique la manera en que emerge Los Espinos (Jarquín, 2004).

El patrimonio hacendario también está circunscripto a una política administrativa escoltada por una estrategia territorial, con la finalidad de convertirse en geosímbolo, a modo de una referencia geográfica de poder. Esta concepción relaciona la génesis de la hacienda con el establecimiento de la familia Jasso. Los cargos políticos documentados de las generaciones de este linaje dan sustento a la proposición de que quien tiene el poder político conserva el poder sobre el territorio (Giménez, 1996).

En este escenario, el protagonismo de la posesión territorial de Los Espinos es interpretado por la familia Jasso, originaria de la capital de la Baja Navarra, villa de San Juan Pie del Puerto, que para el siglo XV pertenecía a España.
Ellos conservaban un cargo noble, preminente y propio de hijosdalgos en su Palacio de Armería en San Pelay, tierra de Cisa, entre los vecinos de la vieja Navarra. Inclusive poseían un escudo de armas, que se hizo constar en la Real Ejecutoria de Nobleza en 1949 (Gónzalez, 1976).

Se hacen presentes en la Nueva España los conquistadores Juan de Jasso el Viejo y su sobrino homónimo Juan de Jasso el Mozo, en arribos de 1524 y 1527, respectivamente (Gónzalez,1976). Los dos, allegados a Hernán Cortés en sus aventuras de conquista, dictaron la presencia de su descendencia en Zamora, iniciando con el capitán Juan de Jasso y Payo el Nieto como vecino de los más antiguos de la villa de Zamora (Rodríguez, 1952).

A partir de entonces se encuentra la cuna cuya estirpe radicó en Zamora con múltiples uniones familiares en generaciones, estableciendo una influencia militar, política y económica predominante en el desarrollo de la villa. Este poder predominó durante 220 años con cargos de regidurías, alcaldías y tenencias registrados desde 1622 , tal como se representa en una línea de tiempo que documenta los actores de la familia desde su presencia en la villa hasta que la hacienda deja de ser patrimonio de los Jasso (ver figura 2). 


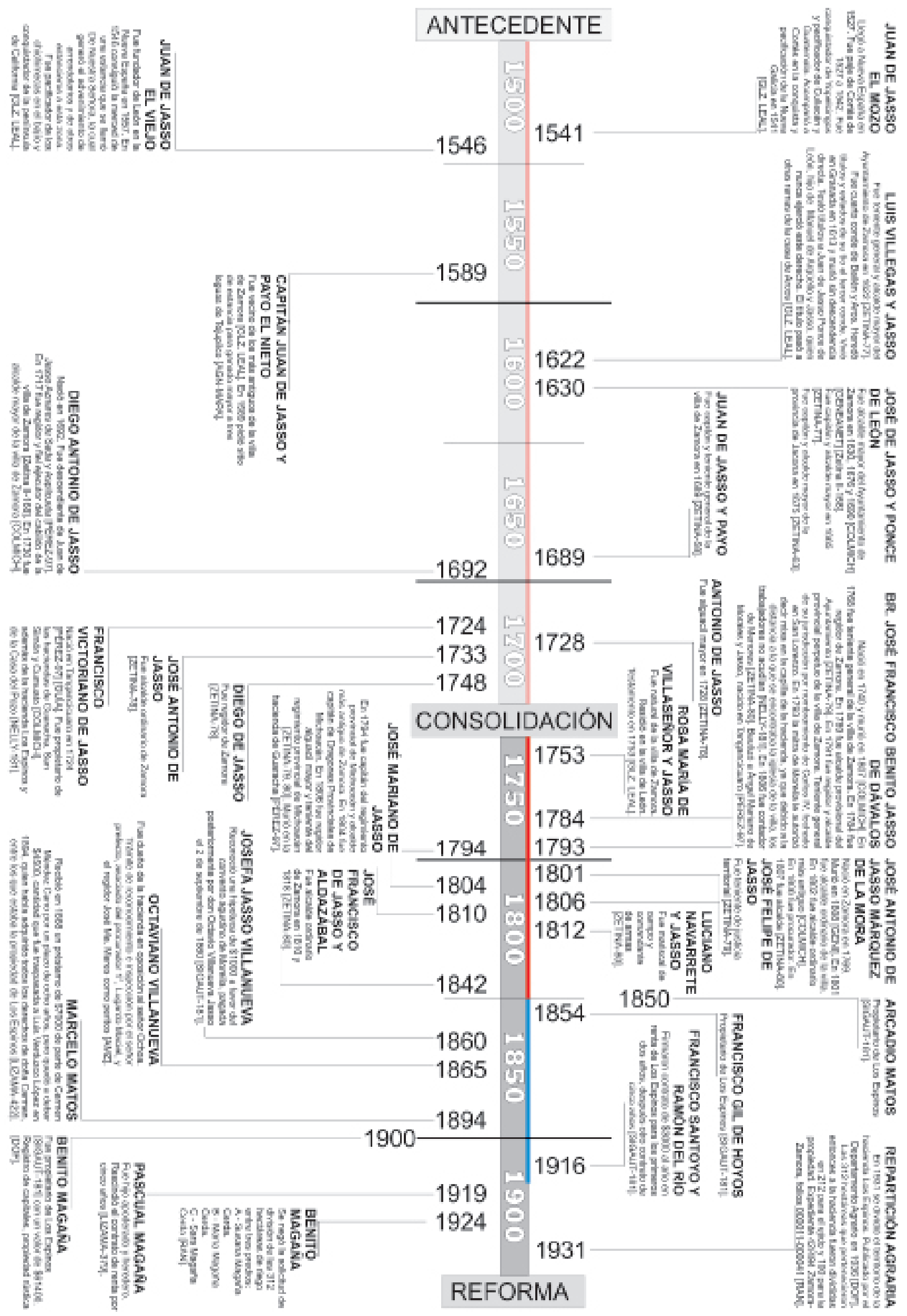

Figura 2. Línea de Tiempo. Integrantes de la genealogía de los Jasso desde su llegada a la Nueva España hasta el último propietario de la hacienda Los Espinos. Autoría propia. 


\section{APOGEO TERRITORIAL}

Las personas de alto poder en la Nueva España tenían la posibilidad de comprar las propiedades de las personas necesitadas y sin recursos ejerciendo su influencia con las autoridades reales. En este sentido, el virrey marqués de Villamanrique derogó algunas de las restricciones para vender por el año de 1588. Más tarde, se dictó la ordenanza al virrey mediante cédula de 1615 por parte del Consejo de Indias para vender en subasta pública mercedes de tierras nuevas, condicionando a los compradores a ratificar sus títulos no reconocidos a la Corona; de otra manera se venderian en dicha subasta al mejor postor. Para los españoles que desearan seguir con la posesión legal de las tierras, existía la posibilidad del pago de una composición moderada. A mediados del mismo siglo, en 1643 , se dispuso que todas las posesiones que no contaran con títulos legítimos fueran consideradas tierras de realengo y, por ende, puestas en subasta pública (Jarquín, 2004).

Este sistema, denominado composición, lo interpreta Jarquín como un rechazo por los propietarios españoles, quienes se oponían a ser investigados por falta de títulos, como era usualmente. De la misma manera, sus influencias de poder se hacian presentes para evitar la medición de sus terrenos; en caso de que ya hubieran sido recompuestos de acuerdo con la ley, reclamaban anular esa disposición. En poco tiempo consiguieron que se expidieran dos cédulas por parte de la Corona: una que omitía la medición de los territorios por medio de una cuota, y otra que amparaba a los dueños de haciendas de prestigio por formar parte de la clase social alta o por ser descendientes de conquistadores.

La hacienda fue la institución económica predominante del Nuevo Mundo y se extendió sobre las tierras deshabitadas y ociosas de ser trabajadas, incluyendo aquellas que pertenecian a las comunidades indigenas e incluso a corporaciones eclesiásticas, entre otras. Los naturales se encontraban amenazados en sus pueblos por las producciones ganaderas, agrícolas y mineras de los españoles, por lo que adoptaron actitudes defensivas. Al recurrir a amparos y demandas proteccionistas para los territorios de las comunidades, se vivió un periodo en que los pueblos fueron agotando sus recursos y liquidando sus bienes en procesos sin solución. El valor de las tierras fue aumentando considerablemente, hasta que estas llegaron a ser el objeto predominante de la colonia. Los que las habitaban estaban obligados a salvaguardarlas, convirtiéndose en algún momento en sus poseedores reales, no necesariamente legales, con lo que surgieron los grandes latifundios.

La hacienda llegó a tener un éxito económico extraordinario, aun sin la inclusión de la comunidad indigena. A pesar de esto, la hacienda evolucionó utilizando el conocimiento milenario de la agricultura de los nativos con sus procedimientos particulares en el tratamiento de las plantas, de la tierra y del agua, incluso en el empleo directo e indirecto de su mano de obra casi ilimitadamente.

De este modo, los territorios concedidos a naturales y colonizadores durante los siglos XVI y XVII mediante mercedes reales fueron adquiriendo diferentes atributos. Las de los indios conservaron sus facultades para las comunidades públicas, a diferencia de las de los españoles, que adoptaron atribuciones de propiedades privadas, lo que originó la composición de extensiones territoriales extraordinarias (Jarquín, 2004). 
A mediados del siglo XVII, dichas composiciones asumieron su punto culminante cuando los dominantes de las tierras recibieron de forma definitiva mercedes de las propiedades que habian usufructuado con títulos irregulares o por tradición familiar, lo que permitió establecer con legalidad y precisión los límites territoriales.

En consecuencia, con el pasar de los años se propiciaron las buenas relaciones entre hacendados y munícipes, y se documentó sobre los abusos que daban cabida a los entendimientos establecidos entre grupos de mando y poder, según lo denunciaba en sus escritos el subdelegado de la Intendencia, Eugenio Fernández Barrera. A su llegada a Zamora el 15 de abril de 1795, hizo valer su poder de gobernante sobre las excentricidades y pretensiones de los individuos y los grupos implicados en corruptelas territoriales, y describió a la villa como el comercio del laborío del campo (Moreno, 1989).

Los principales hacendados eran miembros de los ayuntamientos. En ese momento Francisco Benito Jasso era el propietario de la hacienda Los Espinos, además de ser el integrante de mayor influencia. Su hijo, José Antonio, fue más adelante alcalde ordinario y el más antiguo de Zamora. Considerando las anteriores dinámicas de apropiación territorial del siglo XVII, en alguno de estos casos se hizo posesión de este territorio hacendario, tal como se documenta en un mapa del archivo Manuel Jasso (ver figura 3). 


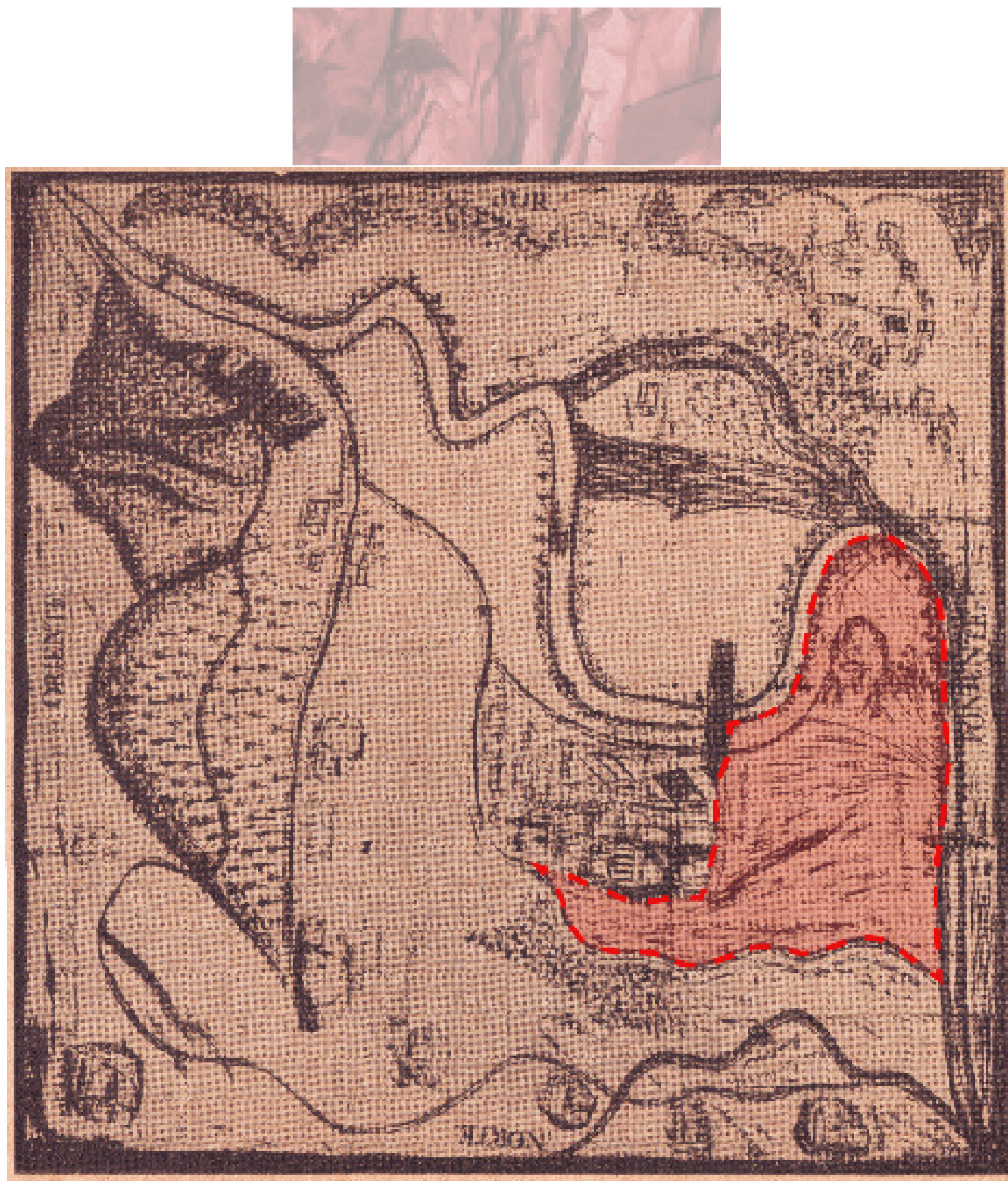

Figura 3. Croquis del valle de Zamora.

- Territorio de la hacienda. Autor Anónimo, Fecha estimada: 1700. Archivo Manuel Jasso en Zamora, apud. Rodríguez Zetina, Arturo. Zamora, ensayo histórico y repertorio documental, p. 14. 
Para vísperas del conflicto de corruptelas, la hacienda de Los Espinos se encontraba en pleno auge, con un respaldo sólido que fortalecía su desarrollo desde diversos ámbitos. Un par de años atrás, en 1795, Benito había pedido autorización a la mitra de Morelia para decir misa en la capilla de la hacienda, ya que los trabajadores no asistían los días de obligación a la capilla de la villa, debido a la lejanía de esta propiedad (Rodríguez, 1952).

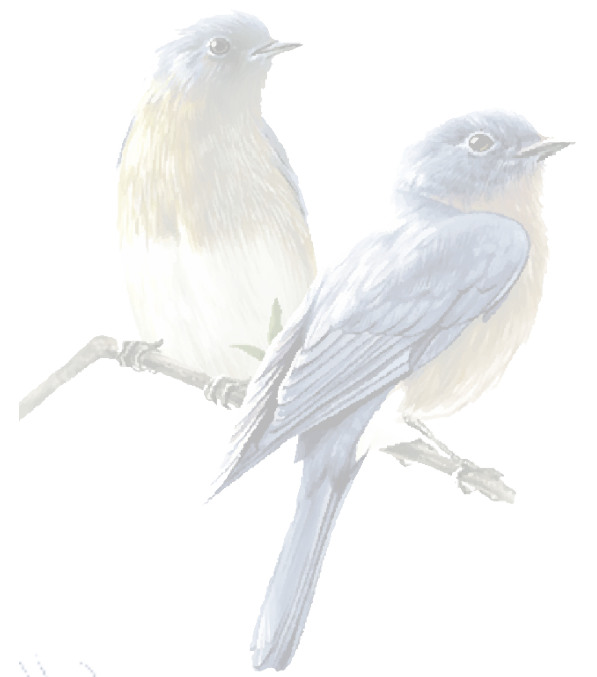

Años más tarde, se presentó una disputa por la existencia de una vertiente pluvial necesaria para el riego de los sembradíos de caña dulce de Los Espinos, con el argumento de que los terrenos de cultivo de las haciendas de Chaparaco, San Juan Bautista, Tamándaro y demás tierras de repartimiento alrededor de la villa contaban con acequias que suministraban el recurso hidráulico para sus riegos y consumos. Tal vez no eran suficientes los recursos del río Duero que rodeaban el sur y el poniente del territorio hacendario. Es posible identificar lo que para entonces fueran las casas y solares dentro de la hacienda, las cuales probablemente pertenecían a los trabajadores y caciques; la casa grande, con su ingenio y su trapiche; además del acceso y su puente directamente conectados al sudoeste de la villa (ver figura 4). 


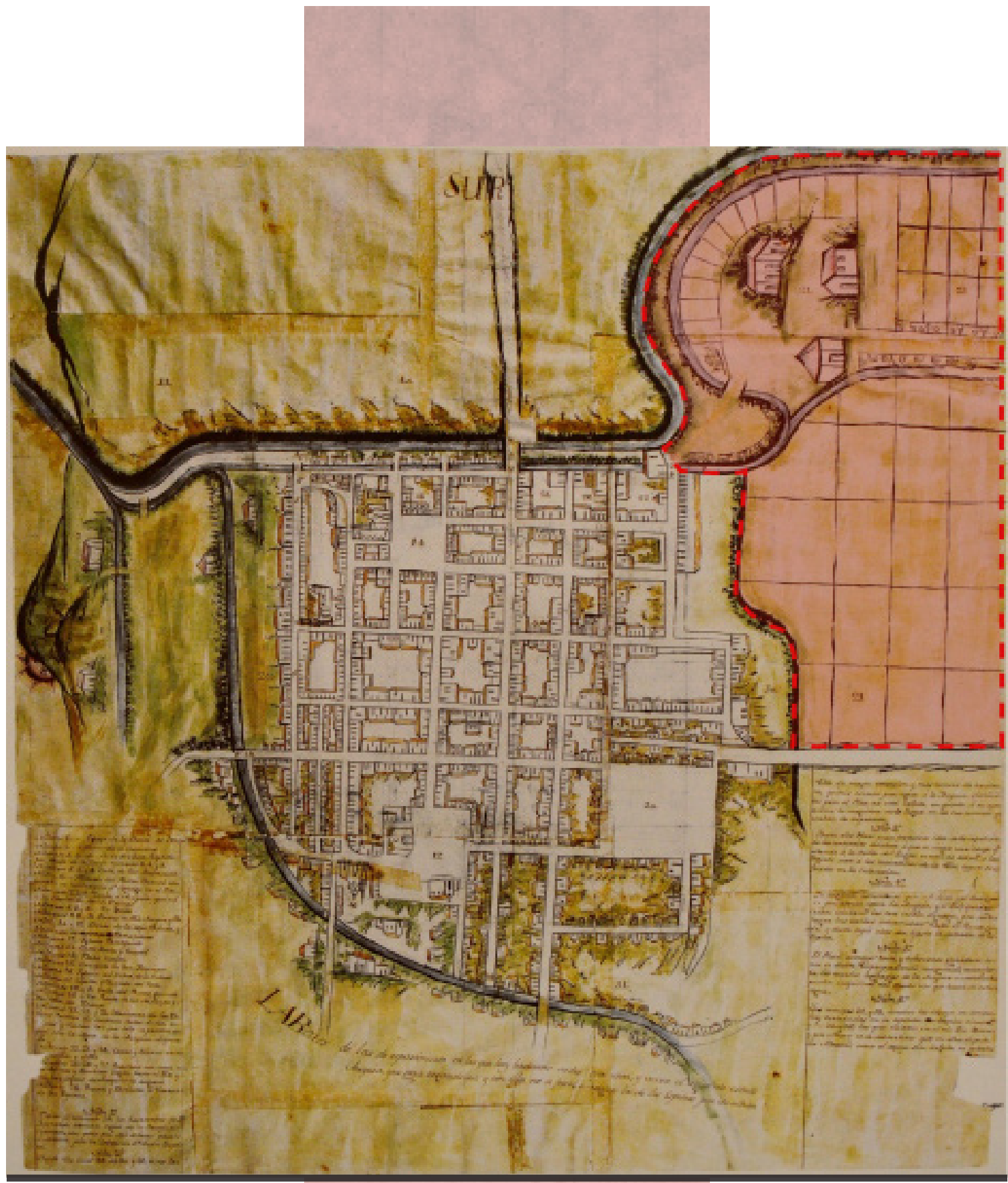

Figura 4. La hacienda los Espinos y la villa de Zamora.

Hacienda Los Espinos. Autor anónimo. Fecha: 1809.

Los escritos del mapa señalan al oriente el cerro de La Beata y a su costado la hacienda de Chaparaco, enseguida la de San Juan Bautista y al sur la de Tamándaro. Ubican lo que sería entonces la parroquia de la villa, el convento de San Francisco, la iglesia del Señor de la Salud, al mismo tiempo que la capilla y plazuela del Teco. Además de mostrar otros sitios importantes de Zamora, el mapa incluye una detallada descripción de las redes fluviales conformadas por zanjas, acequias y ríos, así como su aprovechamiento en las labores agrícolas y habitacionales. Los escritos principalmente argumentan la necesidad de la existencia de la acequia para suministrar de agua a las tierras y vecinos de Los Espinos.

Zamora, Michoacán, Tierras: vol. 2430, exp. 2, f. 59, Archivo General de la Nación. 
En efecto, el escenario de esta época se contextualiza con un poder influyente de la familia, integrado del poder eclesiástico al político. En años posteriores, en los Jasso se desarrolló una importante actividad religiosa. Ángel Mariano José Ignacio de Morales y Jasso, bautizado por su tío Francisco Benito, llegó a ser dignidad de la santa iglesia catedral de México y gobernador de su obispado, antiguo obispo de Sonora, individuo del consejo de Gobierno de la república, asistente al sacro solio pontificio y obispo de Oaxaca. Murió el 27 de marzo de 1843 (Pérez, 1888).

En esta misma época entró en escena la adquisición de una de las haciendas más prósperas del occidente de Michoacán. La hacienda de San Juan Guaracha, en la actual comunidad de Emiliano Zapata, municipio de Villamar, se originó de una de las mercedes otorgadas en 1569 al español Simón Díaz para fundar dos puestos de ganado mayor; allí se dio el primer paso para la conformación del núcleo poblacional regido por este patrimonio hacendario (Montes, 2015).

Cabe mencionar que este complejo llegó a conformar un imperio constituido por varias haciendas lacustres en la región, entre las que se encontraban las haciendas de Buenavista, San Simón, Guarachita y el Valenciano. Sin embargo, la Guaracha formó parte del patrimonio familiar de los Jasso desde su administración por parte del capitán José de Jasso en 1709 estando en posesión de la familia Salceda. Posteriormente fue rematada en 1790 por 209,000 pesos a Victorino Jasso, quien protagonizó una de las etapas más fructíferas del complejo hacendario. Sus herederos militares José Mariano y Diego Moreno de Jasso, dragón de plaza y capitán de Dragones Provinciales de Michoacán con base en Zamora, respectivamente, siguieron preservando esta propiedad al mismo tiempo que el tío Francisco Benito Jasso conservaba Los Espinos (Moreno, 1989).

Es ineludible que las relaciones de ambos patrimonios hacendarios de origen cercano protagonizaron una etapa muy importante del auge de la hacienda en manos de la familia. Aunque la mayoría de las atenciones productivas y desarrollos económicos se concentraban en las haciendas de la ciénega de Chapala, gran parte de la política dentro del periodo de la Independencia se cocinaba en Zamora, donde la familia Jasso intervenía en la planeación de las recetas, "los Jasso y los De la Mora, los nuevos dueños de los latifundios de Guaracha y Buenavista, también fueron ascendiendo gradualmente a las esferas del mando regional" (Moreno, 1989, p. 262). El escenario político, económico y hasta religioso en Zamora lo ocupaba el bachiller Francisco Benito de Jasso, tío del obispo Ángel Mariano, quien posteriormente pasó sus poderes a su hijo José Antonio (Moreno, 1989). 


\section{DECADENCIA}

Este poder político fue reforzado con Diego Moreno de Jasso, primo y contemporáneo de Ángel Mariano. Nació en 1790 en Zamora y fue heredero de la Guaracha. Participó en la guerra de Independencia con los realistas, e inclusive llegó a ser comandante del batallón de infantería del Ejército federal republicano, emplazado en Zamora. Un año más tarde fue nombrado gobernador constitucional de Michoacán, cargo que ostentó hasta 1833. Fue senador en 1840 y diputado al Congreso General en 1843 y 1846.

Entre estos poderes también se afanó el militar, pues los Jasso, fieles a España hasta el Virreinato, también fueron protagonistas del undécimo regimiento de Dragones Provinciales de Michoacán en la plaza de Zamora, durante la Independencia. José Mariano Jasso apareció en listas para enero de 1795 , fue capitán hasta 1804 , ya como alcalde ordinario de primera elección desde 1801.

La familia tuvo diversos problemas durante la insurrección independentista, debido a la comunión con los realistas; las consecuencias de la guerra repercutieron en incendios y ultrajes de sus propiedades en Zamora, Jacona y Tangancícuaro, además de aprehensiones y muertes de los clérigos, como el vicario José Benito y el cura José Antonio Jasso. 
El poder documentado de la amplia parentela de los Jasso, desde su llegada en la conquista hasta esas épocas, expone el poder suficiente para el dominio del territorio acumulado en numerosas regiones. La unión social de hacendados, cabildantes, militares, mercaderes y eclesiásticos fue reducida por los problemas que se presentaban durante la época, con lo que culminó una etapa productiva para la hacienda de Los Espinos.

En la época del Porfiriato, se concluye que la familia Jasso, en unión con los Dávalos, protagonizó la formación de uno de los territorios hacendarios más extensos de Zamora en uno de los periodos finales del latifundio. En relación con esto, Los Espinos pasó de ser un patrimonio hacendario de producción agrícola a constituir un valor financiero en garantías de movimientos económicos, y se convirtió en un elemento más en incremento de riquezas que empoderaban a las familias de élite.

Concretamente, el grupo familiar formado por Francisco, Nicolás, Arcadio, Luis y Francisco Dávalos Jasso, herederos de Nicolás Dávalos y Tompes, vivió la desaparición de una riqueza familiar de abolengo durante la segunda mitad del siglo XIX, lo que desencadenó la propia pérdida de importancia económica de la región, experimentando los padecimientos de las pestes y conflictos familiares que posiblemente fueron factores que provocaron la desintegración.

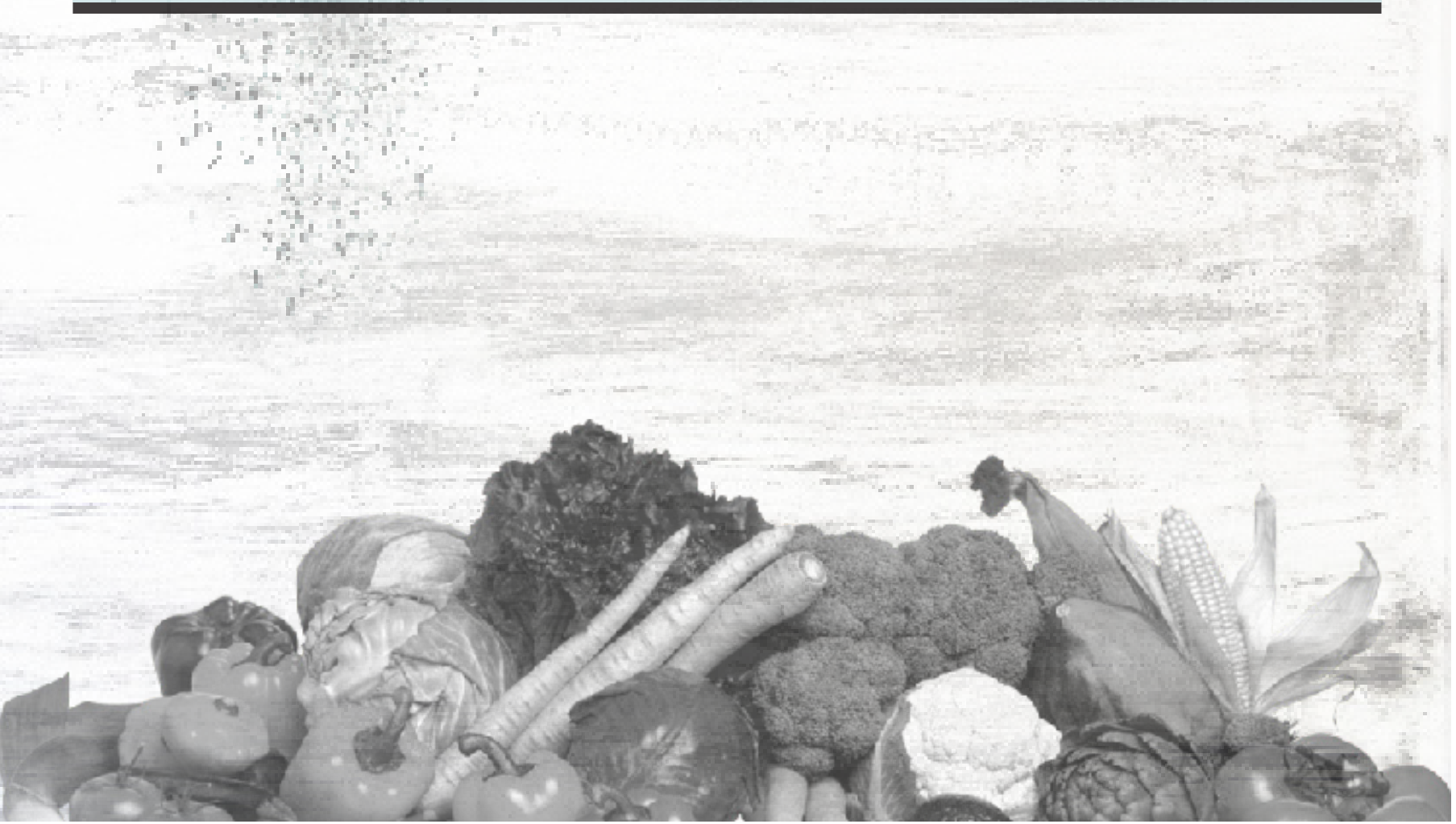




\section{CONCLUSIONES}

Son amplioslos paradigmas en la historia que carecen de evidencias debidamente documentadas. En el mapa del siglo XVI es posible estimar la ubicación de Los Espinos antes de su asentamiento, mientras se establecían las mercedes en los primeros años de la fundación de la villa de Zamora,ya con la limitante de la primera traza del asentamiento español y la barrera natural que conforma el río Duero.

Por otra parte, el mapa del siglo XVIII manifiesta con claridad la trayectoria del río donde se señala a la hacienda, contenida en un contorno que comprende desde la vertiente de riego denominada acequia del Calvario, en el norte de la villa. El terreno se extiende hasta el complejo arquitectónico hacendario en el sur, delimitándose a lo largo del mismo río Duero con dirección al poniente, manifestándose como barrera natural limítrofe y generando un territorio que se extiende al oriente hasta llegar a la traza de Zamora (ver figura 3). Esto demuestra hasta dónde llegó el alcance de sus dominios, con lo que la hacienda emitió una concepción territorial primigenia.

Un siglo más tarde, seguramente por mandato del mismo propietario de la hacienda, se presentó un mapa más detallado con la finalidad de solucionar la disputa de un ramal del río para riego de una parte de los sembradíos de la hacienda y la escasez del recurso que padecían los habitantes de la villa en ese extremo. En este caso, la extensión territorial se seguía limitando por las mismas barreras naturales fluviales, con la variante de que para entonces ya estaba consolidado el asentamiento de San Francisco de los Tecos (ver figura 4). En la pugna condicionada por los agustinos de Jacona, basada en la cédula real de Felipe II que prohibía el asentamiento de órdenes regulares a una distancia menor de seis leguas entre sí (Sigaut, 1991), se detenía un establecimiento oficial de la orden franciscana en la primigenia villa de Zamora. Sin embargo, posteriormente los historiadores mencionan la presencia del asentamiento indígena de los tecos encabezado por la orden de San Francisco en la segunda mitad del siglo $\mathrm{XVII}^{1}$, precisamente en el espacio que se limitaba al norte con la acequia del Calvario y el primer cuadro de la villa, tal como lo demuestra la extensión territorial marcada en el gráfico del siglo XVII (ver figura 3). Para este cambio no hay evidencias que aseguren si dicha extensión fue donada, vendida o expropiada. Sin embargo, esto denota una pérdida territorial precedida de una extensión poblacional.

El territorio hacendario que perteneció a la familia de los Jasso comparte estrechos linderos con Zamora. A simple vista, por la cercanía al primer cuadro de la villa, se podría decir que la ciudad tuvo su origen en este asentamiento productivo, donde su población se desarrolló a partir de una comunidad de labores agrícolas, como es el caso de varias haciendas

1 Es posible que para estos años (1681) ya existiera la primera capilla del Señor de la Salud, mencionada en un testamento de 1686 y que posiblemente atendiera al hospicio del barrio de los Tecos, que si bien no muy distante, mantenía aún una cierta separación de la villa de los Tecos (Sigaut, 1991). 


\section{CONCLUSIONES}

como la de Guaracha, La Sauceda y Buenavista, por mencionar algunas de la región. Sin embargo, el origen del territorio hacendario de Los Espinos fue diferente, pues a pesar de la cercanía de la hacienda, ahora inmersa en la ciudad, los principios mencionados anteriormente hacen distintivo el umbral de este patrimonio al ser posterior a la fundación de Zamora.

El estar tan ligada la hacienda a la villa dio como resultado que las viviendas de los propietarios y de los trabajadores dentro de Los Espinos se limitaran en una estancia eventual. Por esta cercanía, gran parte de los obreros debieron residir en la villa. Por lo que concierne a la familia, se documenta otra propiedad denominada la Casa del Pozo, una de las principales de la ciudad, la cual se ubicaba al poniente de la plaza de armas en el primer cuadro de la traza de la villa. La finca fue destruida en 1950. Se ha dicho que esta propiedad existía desde la fundación de Zamora (Rodríguez, 1952). También se afirma que primitivamente fue el casco de la hacienda Los Espinos; inclusive se alude a una tradición que la menciona como la antigua casa municipal cedida por parte de la familia al ayuntamiento a cambio de ciertas concesiones (Sigaut, 1991), probablemente por los oficios de funcionarios públicos que ejercian entonces los integrantes de la familia. Sin embargo, estos temas ya se han refutado, pero nos ayudan a entender la importancia que tuvo la hacienda durante el desarrollo de la villa en la colonia, la gran relación que existió con esta y la extensión territorial que llegó a alcanzar (ver figuras 3 y 4).

Los hechos que afectaron al territorio que dominó Los Espinos fueron consecuentes a su desintegración gradual a través del tiempo desde sus inicios, inclusive en época independiente, así como en su depresión y transformación en épocas revolucionarias agrícolas, hasta el riesgo de su extinción en nuestros días.

Después de entender lo anterior y al no encontrarse registro fehaciente que dicte el origen de la hacienda en cuestión, este artículo toma el curso con base en los Jasso, antes estudiados, familia oligárquica de la que se tiene bastante registro y se vincula directamente con Los Espinos en el apogeo de su producción como ingenio azucarero del siglo XVII, producto de una merced consolidada.

Existe la primicia de que una élite de hacendados prosperó en la época del Porfiriato, sin dejar a un lado el incremento de riquezas de los imperios pretéritos de abolengo, donde juntos transformaron y modernizaron las haciendas antiguas para adaptar tecnologías emergentes de la época y de esta manera consolidar nuevos complejos hacendarios. Sin embargo, el caso de Los Espinos no tuvo este giro común de sus pares contemporáneos; su marca particular declinó a causa de las decadencias del poder y fortuna de sus dueños, lo que hizo evidente que el declive de la familia hacendaria llevó a la depresión los territorios de su propiedad. 


\section{REFERENCIAS}

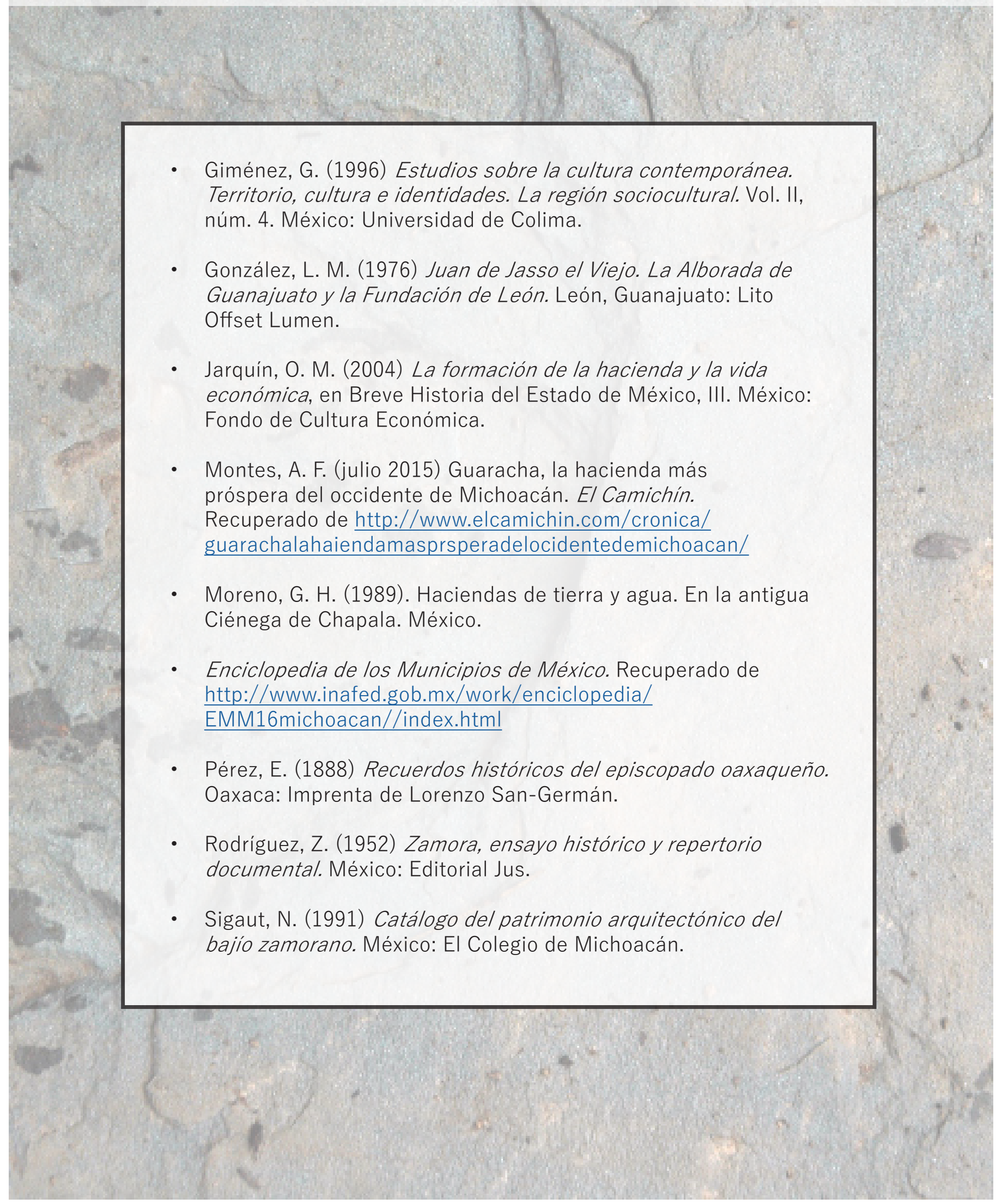




\section{REFERENCIAS DE LA LÍNEA DEL TIEMPO FIGURA (2)}

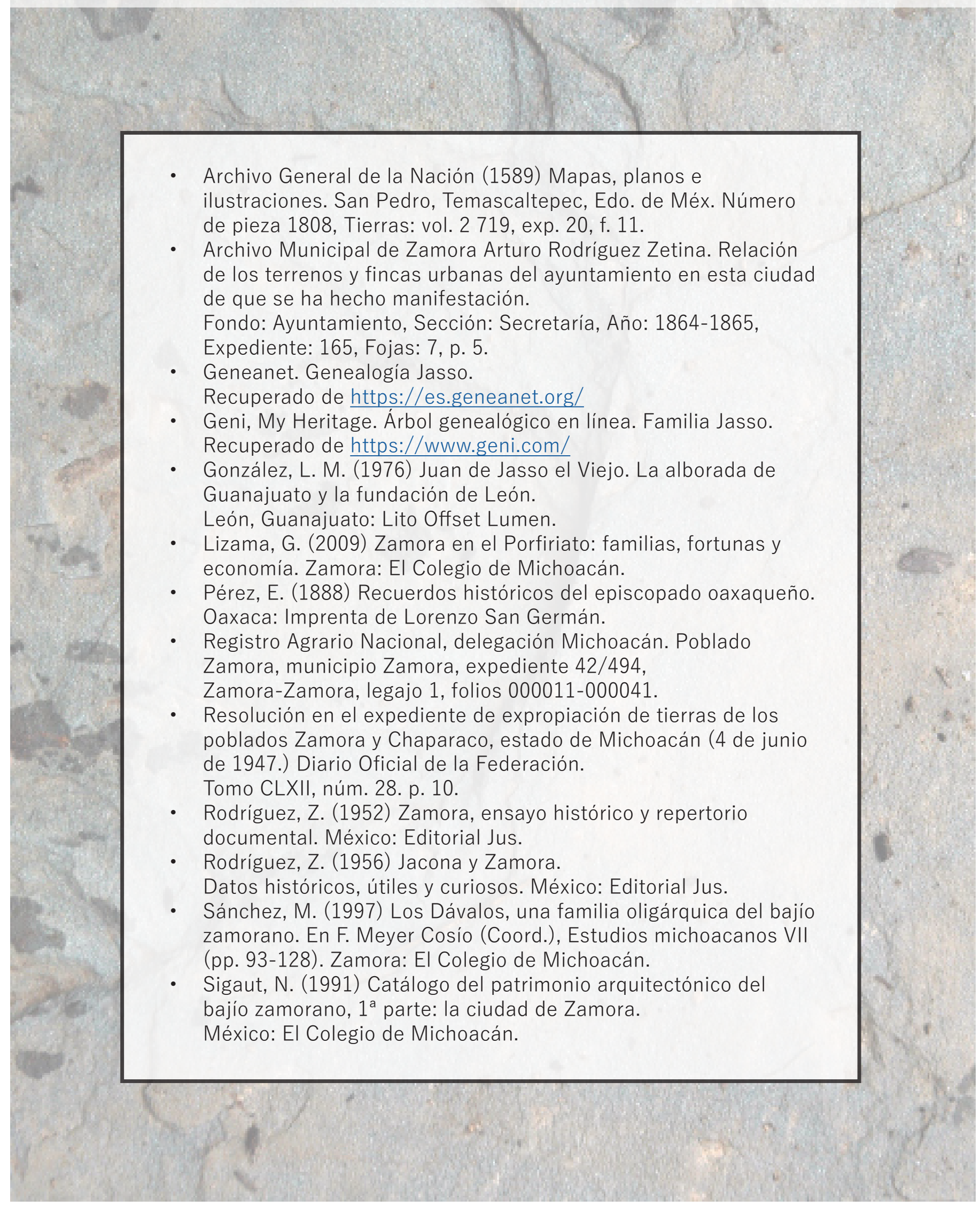



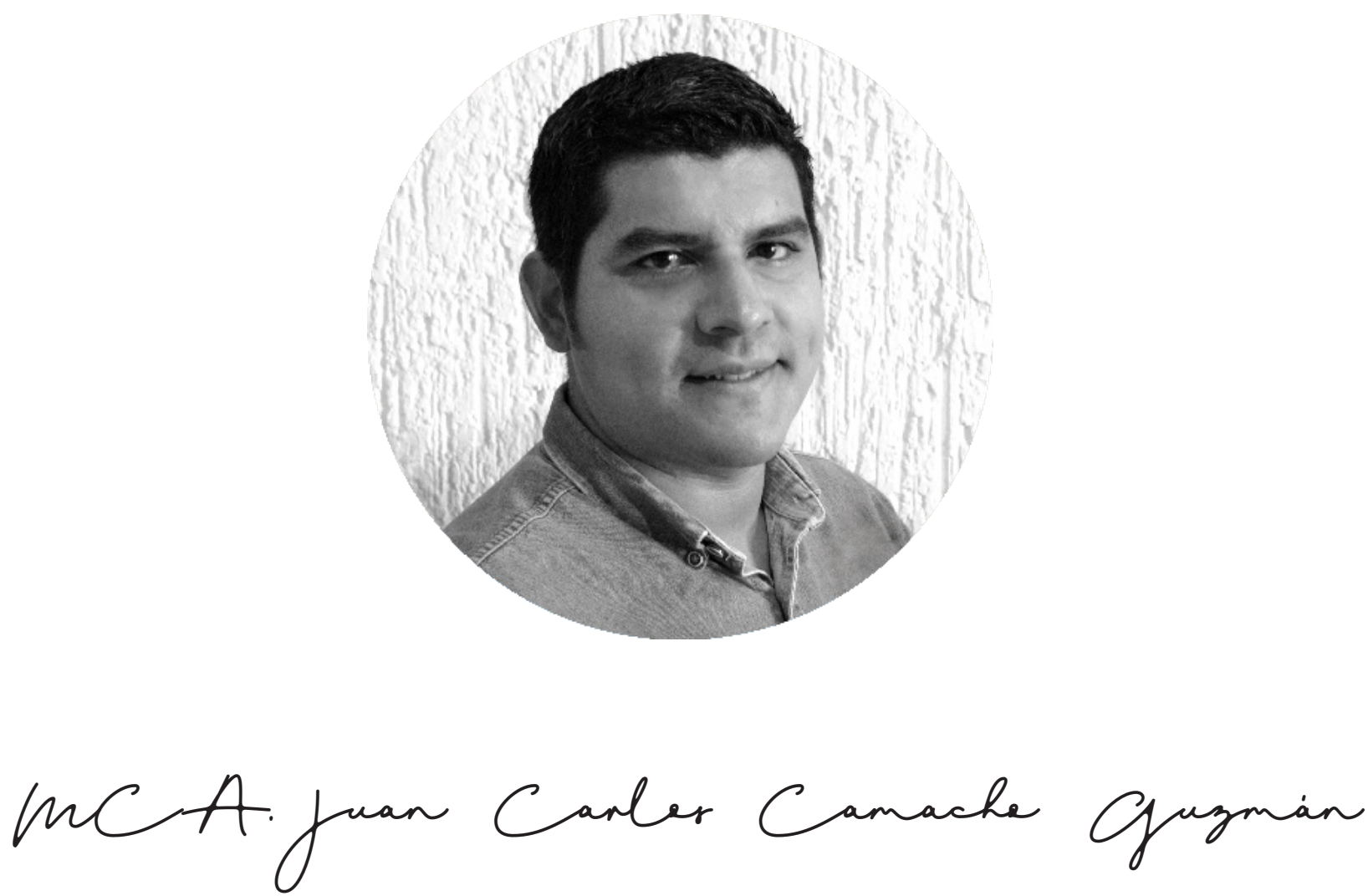

Licenciado en Arquitectura por la Universidad Vasco de Quiroga de Morelia. Maestro en Ciencias de la Arquitectura (PNPC) con orientación a la Conservación de Patrimonio Edificado por la Universidad de Guadalajara. Interventor de obra de conservación en la Rehabilitación de la Imagen Urbana del Bosque Cuauhtémoc de la Ciudad de Morelia en 2011. Interventor de obra de restauración en el Mejoramiento del Entorno Edificado de la calle Valladolid del Centro Histórico de la ciudad de Morelia 2012.

Interventor de obra de integración y restauración en el Proyecto de Revitalización y Reacondicionamiento del Mercado en el Atrio de San Agustín del Centro Histórico de la ciudad de Morelia en 2014.

Actualmente Jefe de la Unidad de Mantenimiento de las sedes Artes Plásticas y Música, y Profesor del Departamento de Proyectos Arquitectónicos de la División de Diseño y Proyectos, del Centro Universitario de Arte, Arquitectura y Diseño, Universidad de Guadalajara.

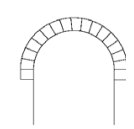




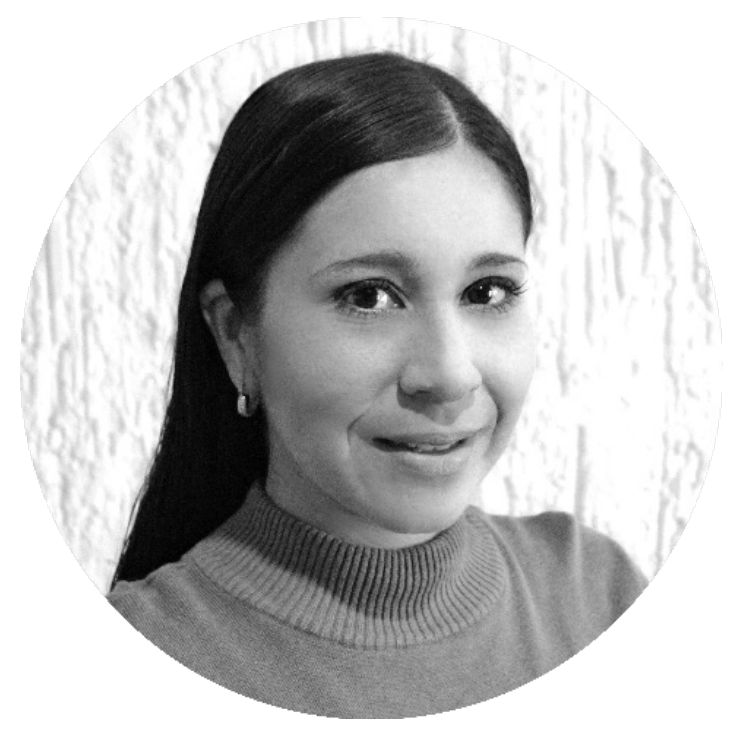

\section{MCST. Jessica Lizet Bautista Hernández}

Licenciada en Nutrición y Maestra en Ciencias de la Salud en el Trabajo por la Universidad de Guadalajara.Participación en cursos y Diplomados en Nutrición Deportiva, estética, auriculoterapia.

Congresos de Innovación en Salud, Psicolingüística y Prevención de riesgos laborales. Co-autora del artículo "Actividad física y salud en el trabajo, gimnasia laboral y pausa laboral activa. Un acercamiento conceptual".

Creadora y responsable del Programa de Disciplina y Salud Integral (PDSI) en el Centro Universitario de Arte, Arquitectura y Diseño. Actualmente Secretario Técnico de Rectoría y Profesora del Departamento de Ciencias Biomédicas, Centro Universitario de Tonalá. 
DIRECTOR

Ernesto Flores Gallo

Centro Universitario de Arte, Arquitectutra y Diseño Benemerita Universidad de Guadalajara, México

\section{CONSEJO EDITORIAL}

Gloria Aslida Thomas Gutiérrez Centro Universitario de Arte, Arquitectura y Diseño Benemerita Universidad de Guadalajara, México

José Alfredo Alcántar Gutiérrez Centro Universitario de Arte, Arquitectura y Diseño Benemerita Universidad de Guadalajara, México

David Zárate Weber Centro Universitario de Arte, Arquitectura y Diseño Benemerita Universidad de Guadalajara, México

Estrellita García Fernández Centro Universitario de Arte, Arquitectura y Diseño Benemerita Universidad de Guadalajara, México

Enrique Solana Suárez Universidad de Las Palmas de Gran Ganaría, España

Luis Ignacio Gómez Arriola Instituto Nacional de Antropología e Historia (INAH) Centro Regional de Occidente, México

\section{COORDINADOR EDITORIAL}

Daniel Rodríguez Medina

Centro Universitario de Arte, Arquitectura y Diseño Benemerita Universidad de Guadalajara

EDITOR RESPONSABLE

Gloria Aslida Thomas Gutiérrez Centro Universitario de Arte, Arquitectura y Diseño Benemerita Universidad de Guadalajara, México

EDITORA ADJUNTA

Atenas Zoe Camila Murillo Muñoz Universidad Virtual de la Universidad de Guadalajara Benemérita Universidad de Guadalajara, México

SECRETARIA TÉCNICA

Gloria Gabriela Alemán Aceves Centro Universitario de Arte, Arquitectura y Diseño Benemérita Universidad de Guadalajara, México

\section{EDITOR TÉCNICO}

Abel de Jesús Carrasco González Centro Universitario de Arte, Arquitectura y Diseño Benemérita Universidad de Guadalajara, México

\section{EDITORES DE SECCIÓN}

Marcela Sofía Anaya Wittman

Centro Universitario de Arte, Arquitectura y Diseño

Benemerita Universidad de Guadalajara, México

Alejandra Robles Delgado Romero

Centro Universitario de Arte, Arquitectura y Diseño

Benemérita Universidad de Guadalajara, México

\section{Mónica Gómez Zepeda}

Centro Universitario de Arte, Arquitectura y Diseño Benemérita Universidad de Guadalajara, México

Juan Antonio García Ugalde

Centro Universitario de Arte, Arquitectura y Diseño

Benemérita Universidad de Guadalajara, México

\section{ASISTENTE EDITORIAL}

Oswaldo Gabriel Esquivel Gomez

Centro Universitario de Arte, Arquitectura y Diseño

Benemérita Universidad de Guadalajara, México

\section{EQUIPO DE DISEÑO Y CUIDADO DE INTERIORES}

Atenas Zoe Camila Murillo Muñoz

Universidad Virtual de la Universidad de Guadalajara Benemérita Universidad de Guadalajara, México

Gloria Gabriela Alemán Aceves

Centro Universitario de Arte, Arquitectura y Diseño Benemérita Universidad de Guadalajara, México

Abel de Jesús Carrasco González

Centro Universitario de Arte, Arquitectura y Diseño

Benemérita Universidad de Guadalajara, México

\section{COMUNICACIÓN}

Uriel Aceves Jiménez

Centro Universitario de Arte, Arquitectura y Diseño Benemérita Universidad de Guadalajara, México

Omar Arturo Huerta Barba

Centro Universitario de Arte, Arquitectura y Diseño Benemérita Universidad de Guadalajara, México

Sergio Arnoil García Carrillo

Centro Universitario de Arte, Arquitectura y Diseño

Benemérita Universidad de Guadalajara, México

\section{CORRECTOR DE ESTILO}

Punto y Aparte

Asesoría en redacción y ortografía 


\section{COMITÉ EDITORIAL}

Ana Portalés Mañanós

Universidad Politécnica de Valencia, España

María Teresa Palomares Figueres Universidad Politécnica de Valencia, España

Marisol Ordaz Tamayo Universidad Autónoma de Yucatán, México

Luis Alberto Torres Garibay Universidad Michoacana de San Nicolás de Hidalgo, México

Eugenia María Azevedo Salomao Universidad Michoacana de San Nicolás de Hidalgo, México

José Refugio De La Torre Curiel Centro Universitario de Ciencias Sociales e Historia Benemerita Universidad de Guadalajara, México

Juan López García

Centro Universitario de Arte, Arquitectura y Diseño Benemerita Universidad de Guadalajara, México

Marcela Sofía Anaya Wittman Centro Universitario de Arte, Arquitectura y Diseño Benemerita Universidad de Guadalajara, México

Adriana Ruíz Razura Centro Universitario de Arte, Arquitectura y Diseño Benemerita Universidad de Guadalajara, México

Vol. 1 No. 1

\section{Enero - Junio 2019} ISSN EN TRÁMITE

Para mayor información y envio de articulos dirijase a:

Mtra. Gloria Aslida Thomas Gutiérrez

Centro Universitario de Arte, Arquitectura y Diseño Benemerita Universidad de Guadalajara, México

E-mail revista_hcpe@cuaad.udg.mx

\section{Atenas Zoe Camila Murillo}

Centro Universitario de Arte, Arquitectura y Diseño Benemerita Universidad de Guadalajara, México Email zoe.murillo@cuaad.udg.mx 


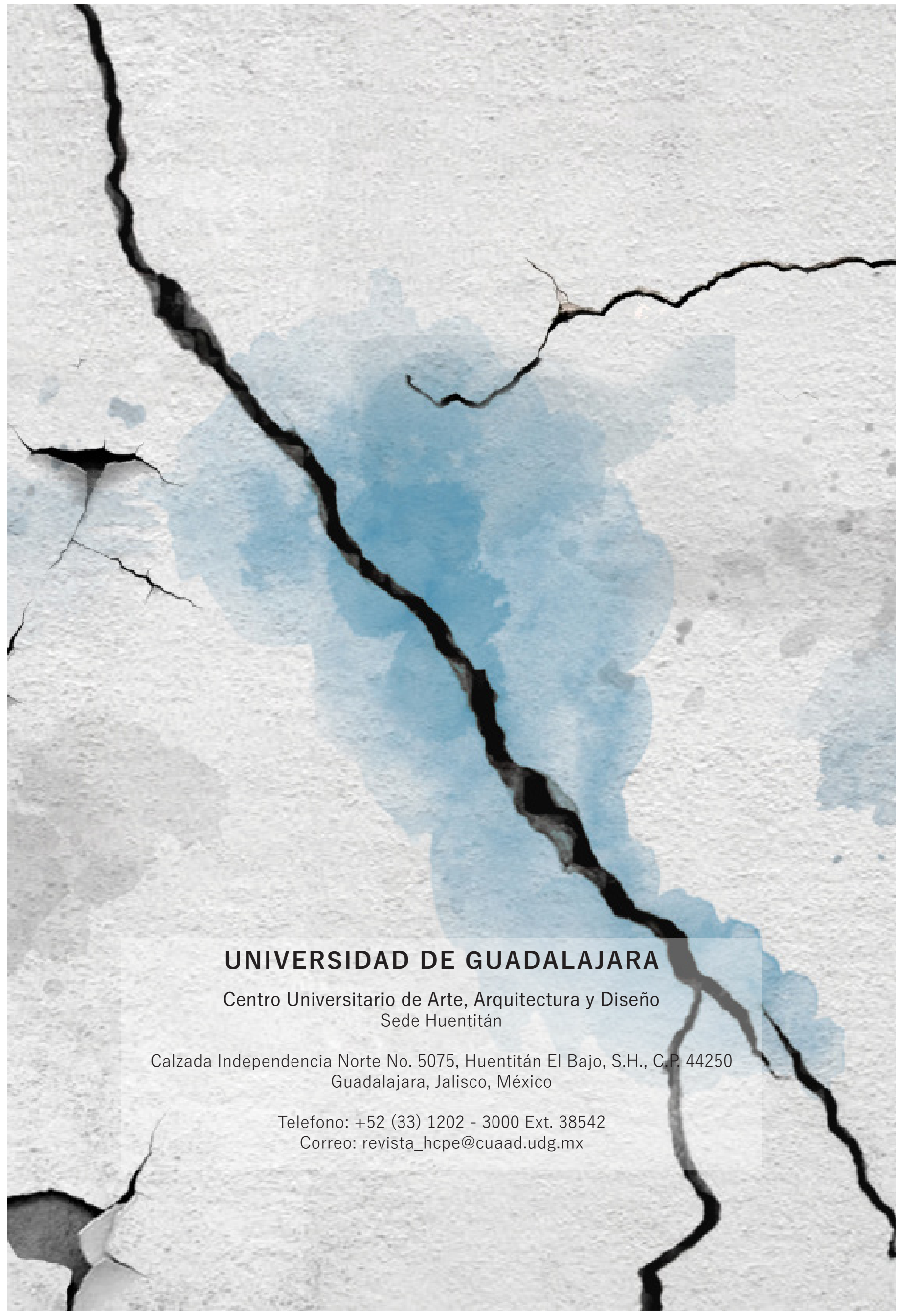

